\title{
The incidence and risk factors for ovarian metastasis and overall survival with ovarian preservation for early-stage adenocarcinoma of the cervix-A meta-analysis
}

\author{
Dongchen $\mathrm{Wu}^{1, \dagger}$, Lihua Zhang ${ }^{1, \dagger}$, Nitish Beharee ${ }^{1}$, Li Yang ${ }^{1}$, Yinan $\mathrm{Wu}^{1}$, Yingchun Wang ${ }^{1}$, \\ Mengmeng Lv ${ }^{1}$, Jin $\mathrm{Lu}^{1}$, Jinhua Wang ${ }^{1, *}$ \\ ${ }^{1}$ Cancer Hospital Affiliated to Nanjing Medical University, Jiangsu Cancer Hospital, Jiangsu Institute of Cancer Research, 210000 Nanjing, Jiangsu, \\ China \\ *Correspondence: wangjinhua588@163.com (Jinhua Wang) \\ ${ }^{\dagger}$ These authors contributed equally. \\ Academic Editor: Enrique Hernandez \\ Submitted: 15 August 2021 Revised: 27 November 2021 Accepted: 30 November 2021 Published: 15 February 2022
}

\begin{abstract}
Objective: To compare the incidence of ovarian metastasis (OM) in early stage adenocarcinoma (AC) and squamous cell carcinoma (SCC) of the cervix, evaluate the overall survival with ovarian preservation and determine risk factors of OM for early stage AC. Data sources, methods of study selection: We searched the Cochranes database, Embase, and PubMed for publications to November 2020. The articles reporting the incidence, risk factors and overall survival of $\mathrm{OM}$ in AC were included. Articles that lacked sufficient data of the odds ratios (ORs) and 95\% confidence intervals (CIs) were excluded. A fixed effects model was used to calculate OR and $95 \%$ CIs. Eggers test and Funnel plot were used to test the publication bias. Forest plots was used to present and synthesise results. Tabulation, integration and results: In the meta-analysis, the incidence of $\mathrm{OM}$ of $\mathrm{AC}$ was higher than that of SCC (OR 5.68, 95\% CI 4.40-7.32, $I^{2}=28.1 \%$ ) in stage IA-IIB. The incidence of OM was $0 \%$ in stage IA, $2.72 \%$ in stage IB, $5.95 \%$ in stage IIA, and $12.86 \%$ in stage IIB AC. Ovarian preservation was not significantly associated with OS (OR $0.53,95 \%$ CI $0.35-0.80, I^{2}=37.8 \%$ ) in early stage of AC. We found seven risk factors for OM: deep stromal invasion (OR 8.80, 95\% CI 3.20-24.23, $I^{2}=0 \%$ ), corpus uteri invasion (OR 6.29, 95\% CI $\left.3.36-11.77, I^{2}=21.8 \%\right)$, tumor size $>4 \mathrm{~cm}\left(\right.$ OR $3.78,95 \%$ CI 1.86-7.69, $\left.I^{2}=30.5 \%\right)$, FIGO stage IIA (OR 3.67, 95\% CI 1.98-6.81, $I^{2}=$ $0 \%$ ), FIGO stage IIB (OR 4.31, 95\% CI 2.74-6.77, $I^{2}=0 \%$ ), FIGO stage II (OR 3.99, 95\% CI 2.49-6.41, $I^{2}=0 \%$ ) and lympho-vascular space invasion (OR 2.90, 95\% CI 1.36-6.17, $I^{2}=0 \%$ ). Conclusions: Ovarian preservation is only recommended in stage IA and stage IB AC without risk factors, but not reasonable for stage IIA and IIB AC. Both stage IIA and IIB are risk factors for OM in early stage AC.
\end{abstract}

Keywords: Adenocarcinoma of cervix; Ovarian preservation; Ovarian metastasis; Risk factors

\section{Introduction}

Secondary to the increase in early screening, the incidence of cervical squamous cell carcinoma (SCC) has decreased while the incidence of cervical adenocarcinoma (AC) is increasing [1,2]. More than 33\% of AC patients were younger than 40 years old [3]. Some studies have found that the incidence of ovarian metastasis (OM) for $\mathrm{AC}$ was higher than that for SCC by $4.5 \%-7.8 \%$ [4-10], but some studies also reported that the incidence of $\mathrm{OM}$ in early AC and SCC were similar [11]. It is still controversial whether young patients with early AC should have ovarian preservation. Studies have shown that people who underwent early oophorectomy had a higher mortality rate if they had not received estrogen therapy $[12,13]$. We identified the difference in the incidence of early AC and SCC and the overall survival (OS) of ovarian preservation for early AC through meta-analysis. We also identified the risk factors of $\mathrm{OM}$ in early AC.

\section{Materials and methods}

\subsection{Search method}

Cochranes database, Embase, and PubMed database were searched to November 2020: "cervical cancer", "adenocarcinoma of the cervix", "ovarian metastasis", and "ovarian preservation" were used as search terms in the title or abstract. The language was limited to "English".

\subsection{Criteria for inclusion and exclusion}

The criteria for inclusion were as follows: (1) The diagnosis of cervical AC. (2) Prospective or retrospective cohort. (3) The sample size greater than 20. (4) Studies reported the incidence of OM for SCC and AC. (5) Studies that included the survival rate of patients with removal and preservation of ovaries for AC. (6) Studies that reported the risk factors for $\mathrm{OM}$ in $\mathrm{AC}$.

The criteria for exclusion were as follows: (1) The sample size was less than 20. (2) Lack of sufficient data of the odds ratios (ORs) and 95\% confidence intervals (CIs). (3) Overlapping or duplicate articles. 


\subsection{Data extraction}

Data extraction was performed by one author and checked by a second author. The information extracted from each study was as follows: author, country, total number of patients, year of publication, FIGO stage, number of patients undergoing oophorectomy and ovarian preservation, incidence of $\mathrm{OM}$.

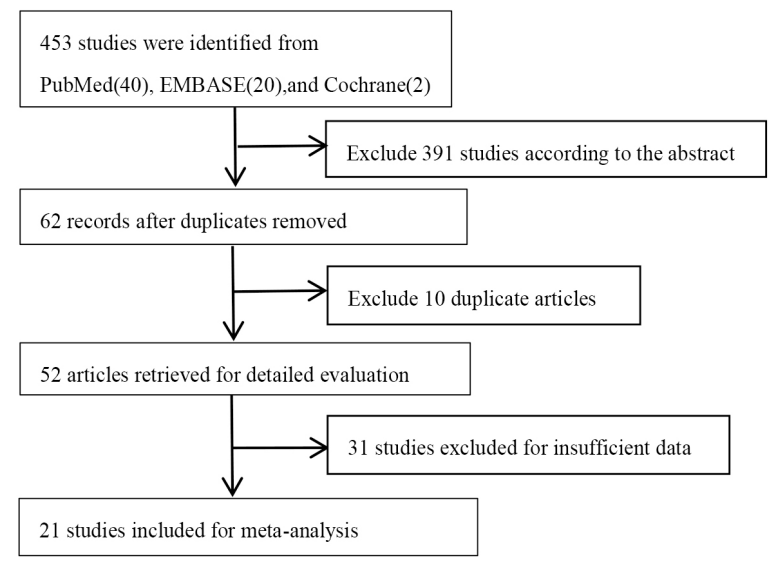

Fig. 1. The flow diagram for selection of literature.

\subsection{Quality assessment}

The quality of the included studies was evaluated independently by the use of Newcastle-Ottawa Quality Assessment Scale. High-quality studies were defined as final score $\geq 6$.

\subsection{Statistical analysis}

Stata 12.0 (STATA, College Station, TX; Computing Resource Center, Santa Monica, CA, USA) was used for the statistics of ORs and $95 \%$ CIs. Heterogeneity was assessed by Cochran's-Q test and $I^{2}$ statistics. Heterogeneity was regarded as statistically significant when $I^{2}>50 \%$ and the $p$-value $<0.05$ in Cochran's-Q test. If needed, a randomeffects model was chosen.

\subsection{Publication bias and sensitivity analysis}

Eggers test was used to assess for publication bias. Publication bias was defined as $p<0.05$. Funnel plot was also used to test the publication bias. Sensitivity analysis was assessed by deleting one study at one time to examine its effect on the final result.

\subsection{FIGO stage}

FIGO 2018 classification was used when abstracting the information from the selected articles. The FIGO stage was adjusted to 2018 classification if the articles were published before 2018 .

\section{Results}

\subsection{Search results and study features}

The flow diagram for literature selection is shown in Fig. 1. Eleven studies were obtained in the meta-analysis for the comparison of the rate of the ovarian metastasis for AC and SCC [4-11,14-16]. Six studies were included in the meta-analysis for the overall survival with ovarian preservation in AC [17-22]. Twelve studies were included for the risk factors of ovarian metastasis in AC [57,9,10,19,20,23-27] (Table 1, Ref. [4-11,14-26]).

\subsection{Quality assessment and publication bias of the included studies}

The score of the Newcastle-Ottawa Quality Assessment Scale is showed in Table 2 (Ref. [4-11,14-26]). For the comparison of the incidence of OM for early AC and SCC, Funnel plot showed a low risk of publication bias (Fig. 2D, Eggers test: $p=0.079$ ). Sensitivity analysis showed no significant change on the final result after one study was deleted (Fig. 2E). For the survival outcome of ovarian preservation, Funnel plot also showed a low risk of publication bias (Fig. 3C, Eggers test: $p=0.625$ ). Sensitivity analysis showed no significant change on the final result after one study was deleted (Fig. 3D). For the risk factors of OM for early stage AC, $p$ value of Eggers test for the included studies were as follows: corpus uteri invasion $p=$ 0.246 , deep stromal invasion $p=0.716$, age $>45 p=0.248$, LVSI $p=0.403$, tumor size $>4 \mathrm{~cm} p=0.536$, tumor grade $p=0.901$, FIGO IIA $p=0.223$, FIGO IIB $p=0.264$, and FIGO II $p=0.213$.

\subsection{Comparison of the incidence of $O M$ in early $A C$ and $S C C$}

A total of 21,466 patients (AC 3711; SCC 17,755) who underwent hysterectomy and oophorectomy could be obtained from the 11 studies. The incidence of OM for AC was higher than that for SCC in stage IA-IIB (OR 5.68, 95\% CI $4.40-7.32, I^{2}=28.1 \%$ ) (Fig. $\left.2 \mathrm{~A}\right)$. In the subgroup, a total of 772 patients with $\mathrm{AC}$ and 3867 patients with SCC from 5 studies were included to compare the incidence of OM for AC and SCC in stage I. A total of 2597 patients (AC 289; SCC 2308) from 4 studies were obtained to compare the incidence of $\mathrm{OM}$ for $\mathrm{AC}$ and $\mathrm{SCC}$ in stage II. The incidence of OM in AC was higher than that of SCC in stage I (OR 8.40, 95\% CI 4.15-17.01, $I^{2}=19.7 \%$ ) (Fig. 2B) and II (OR 7.31, 95\% CI 4.33-12.35, $I^{2}=0 \%$ ) (Fig. 2C).

Overall, the incidence of $\mathrm{OM}$ in the $\mathrm{AC}$ and $\mathrm{SCC}$ group were $3.85 \%$ and $0.68 \%$ respectively. In the $\mathrm{AC}$ group, the incidence of OM was $0 \%$ in stage IA, $2.72 \%$ in stage IB, $5.95 \%$ in stage IIA, and $12.86 \%$ in stage IIB. In the $\mathrm{SCC}$ group, the incidence of OM was $0 \%$ in stage IA, $0.34 \%$ in stage IB, $0.8 \%$ in IIA, and $2.25 \%$ in stage IIB (Table 1). 
A

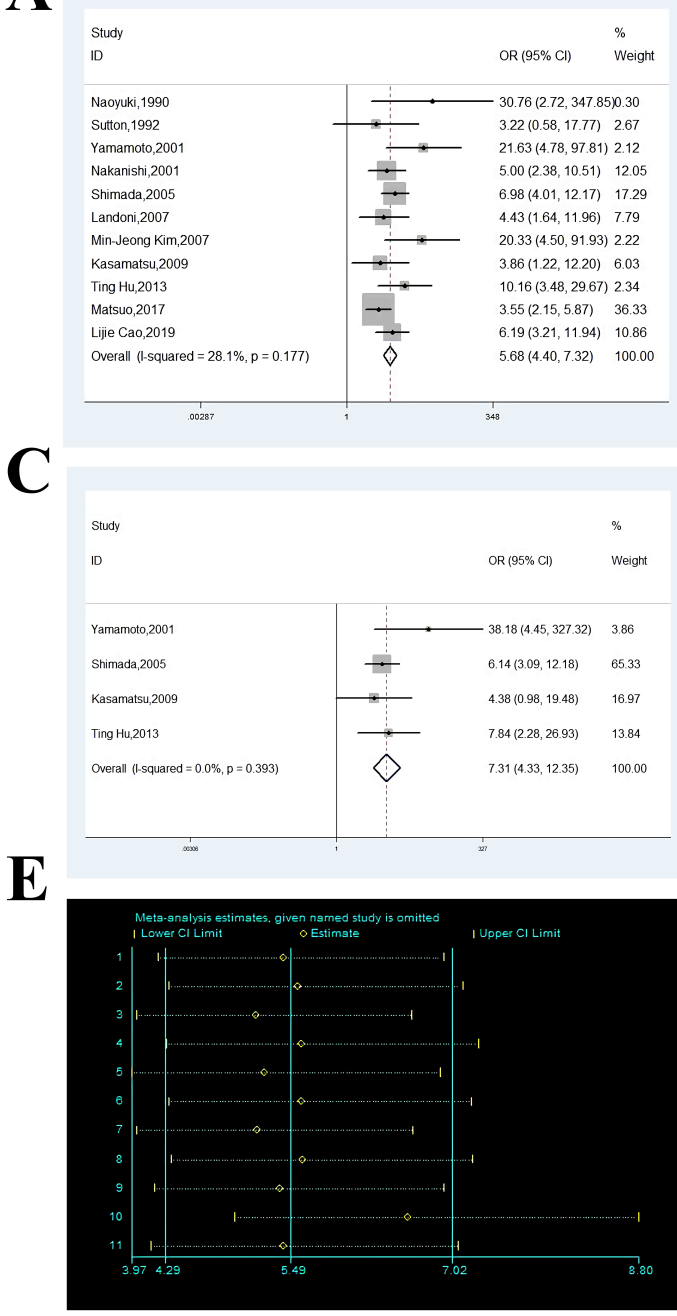

B

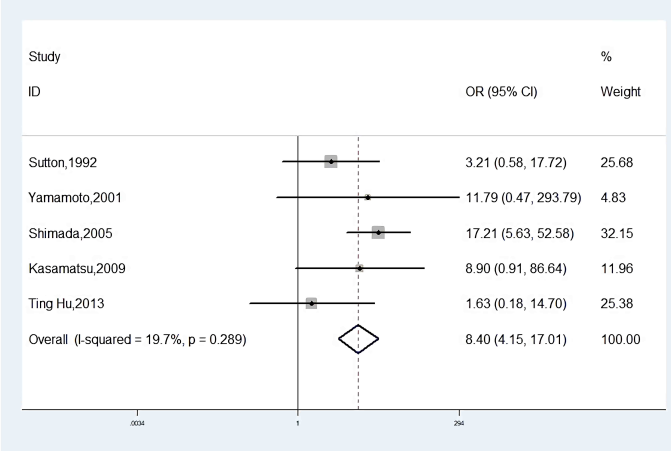

D

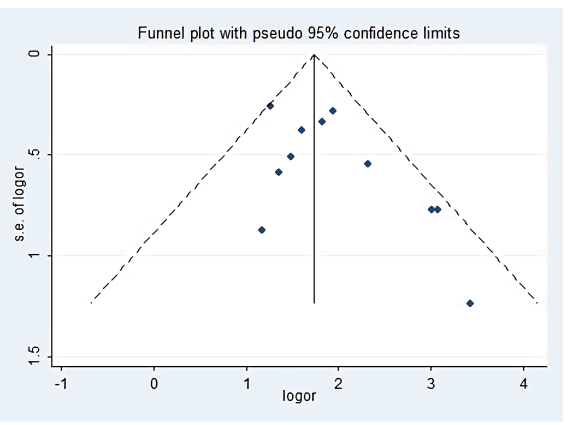

Fig. 2. Comparation of the rate of OM between AC and SCC. (A) Forest plots of comparation of the rate of OM between AC and SCC in stage IA-IIB. (B) Forest plots of comparation of the rate of OM between AC and SCC in stage I. (C) Forest plots of comparation of the rate of OM between AC and SCC in stage II. (D) Funnel plot of the 11 included studied showed a low risk of publication of bias. (E) Sensitivity analysis showed no significant change on the final result after one studies was deleted.

\subsection{Survival outcome of ovarian preservation}

Ovarian preservation occurred in 930 patients while 2493 patients underwent oophorectomy in the 6 studies. Ovarian preservation was not associated with statistically significant OS (OR $0.53,95 \%$ CI $0.35-0.80, I^{2}=37.8 \%$ ) in early stage of AC (Fig. 3A). In the subgroup of stage $\mathrm{I}$, ovarian preservation was not associated with statistically significant OS (stage I: OR $0.46,95 \%$ CI $0.28-0.75, I^{2}=$ $0.7 \%$ ) (Fig. 3B). We did not perform the subgroup analysis in stage II and PFS because of lack of useful data.

Among the 6 studies which included stage IA-IIB AC, the 5-year overall survival rate of patients with or without ovarian preservation was $96.99 \%$ and $94.46 \%$ respectively $(p=0.084)$. For stage I, the 5-year overall survival rate of patients with or without ovarian preservation was $97.57 \%$ and $95.62 \%$ respectively $(p=0.072)$.

\subsection{Risk factors of OM for early stage of $A C$}

A total of 3086 patients with AC were included in the 12 studies. We found seven risk factors for OM: deep stromal invasion (OR 8.80, 95\% CI 3.20-24.23, $I^{2}=0 \%$ ), corpus uteri invasion (OR 6.29, 95\% CI 3.36-11.77, $I^{2}=$ $21.8 \%$ ), tumor size $>4 \mathrm{~cm}$ (OR 3.78, 95\% CI 1.86-7.69, $I^{2}=30.5 \%$ ), FIGO stage IIA (OR 3.67, 95\% CI 1.98-6.81, $I^{2}=0 \%$ ), FIGO stage IIB (OR 4.31, 95\% CI 2.74-6.77, $I^{2}=0 \%$ ), FIGO stage II (OR 3.99, 95\% CI 2.49-6.41, $I^{2}=$ $0 \%$ ) and lympho-vascular space invasion (OR $2.90,95 \% \mathrm{CI}$ $1.36-6.17, I^{2}=0 \%$ ) (Fig. 4A-H). Age $>45$ (OR 0.95, 95\% CI $0.46-1.97, I^{2}=0 \%$ ) and tumor grade (OR $0.93,95 \% \mathrm{CI}$ $0.4-2.18, I^{2}=0 \%$ ) were not the risk factors for OM in early stage AC (Fig. 4I,J). 
A

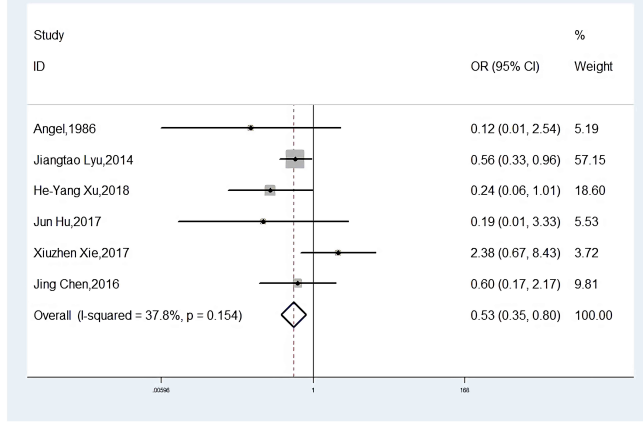

C

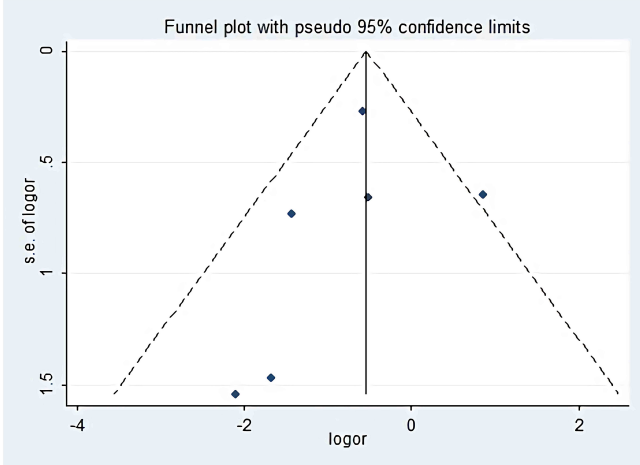

B

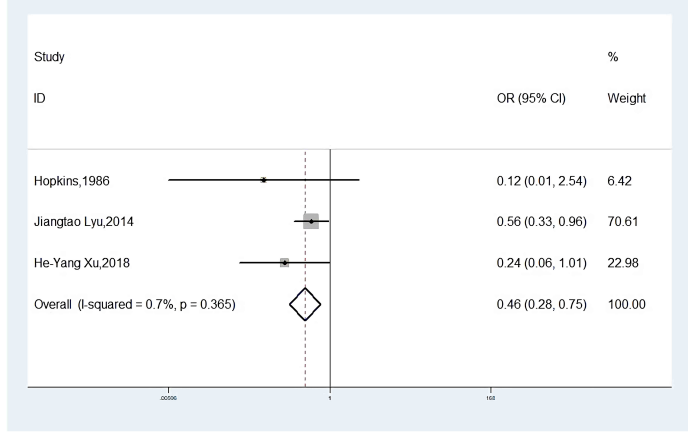

D

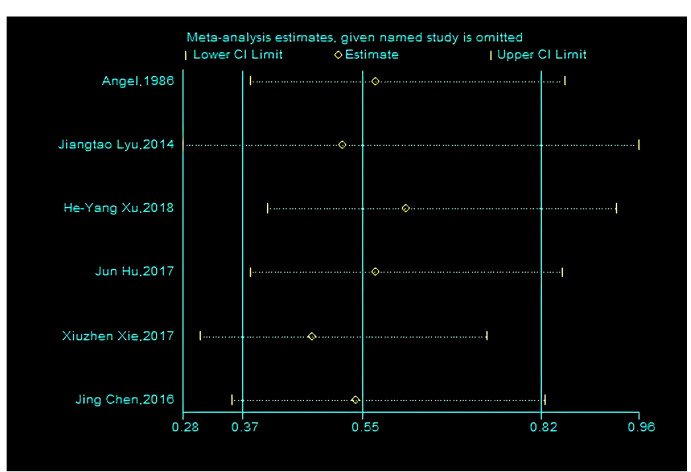

Fig. 3. Oncological outcomes of ovarian preservation for AC. (A) Forest plots of the oncological outcomes of ovarian preservation for stage IA-IIB AC. (B) Forest plots of the oncological outcomes of ovarian preservation for stage I AC. (C) Funnel plot of the 6 included studied showed a low risk of publication of bias. (D) Sensitivity analysis showed no significant change on the final result after one studies was deleted.

A

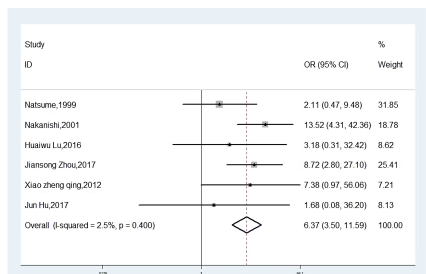

D

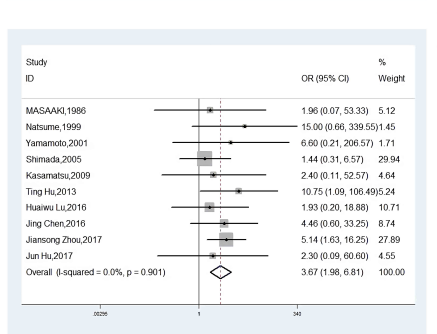

G

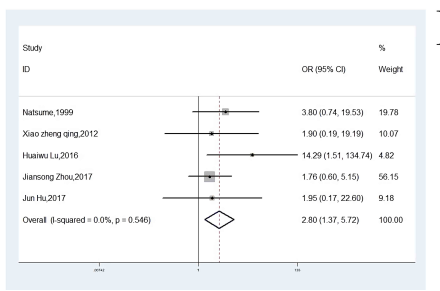

B

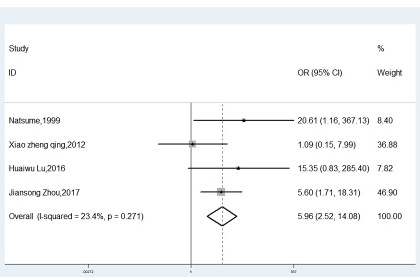

E

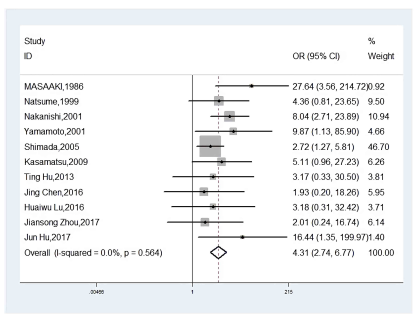

H

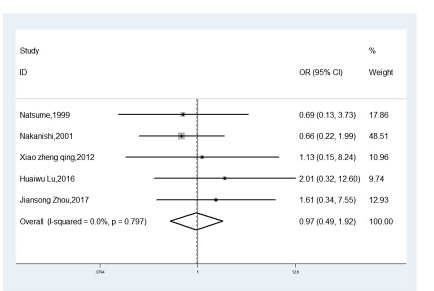

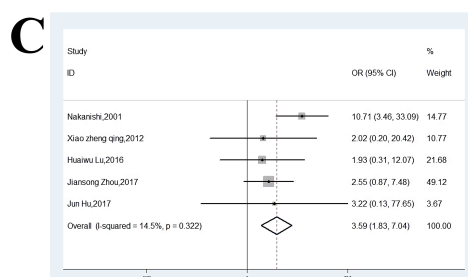

F

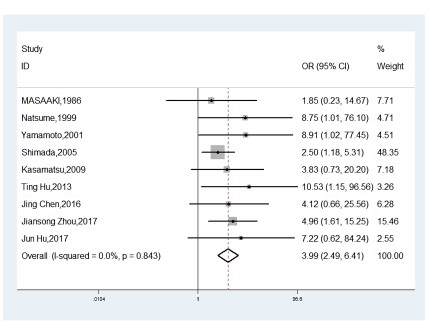

I

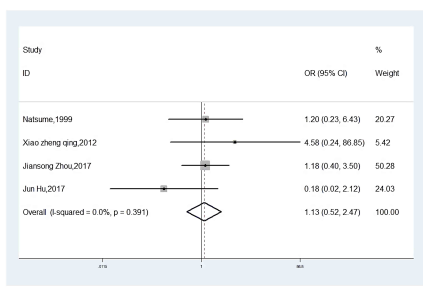

Fig. 4. Forest plots of 7 risk factors of OM for AC. (A) Corpus uteri invasion. (B) Deep stromal invasion. (C) Tumor size $>4 \mathrm{~cm}$. (D) FIGO stage IIA. (E) FIGO stage IIB. (F) FIGO stage II. (G) Lympho-vascular space invasion. (H) Age $>45$. (I) Tumour grade. 
Table 1. Characteristic of the included studies.

\begin{tabular}{|c|c|c|c|c|c|c|c|c|c|c|c|c|}
\hline \multirow{2}{*}{ Study } & \multirow{2}{*}{ Year $\mathrm{C}$} & \multirow{2}{*}{ Country } & \multirow{2}{*}{ yistology } & \multirow{2}{*}{ FIGO stage } & \multirow{2}{*}{$\frac{\text { Patients }}{n}$} & \multirow{2}{*}{$\frac{\text { Ovarian }}{\text { preservation }}$} & \multirow{2}{*}{ Oophorectomy } & \multirow{2}{*}{$y \frac{\text { Rate of OM }}{\text { for SCC }}$} & \multirow{2}{*}{ Stage } & \multirow{2}{*}{$\frac{\text { Rate of } \mathrm{OM}}{\text { for } \mathrm{AC}}$} & \multirow{2}{*}{ Stage } & \multirow{2}{*}{$\frac{\text { Risk factors of }}{\text { OM for AC }}$} \\
\hline & & & & & & & & & & & & \\
\hline Tabata et al. [26] & 1986 & Japan & $\mathrm{SCC}, \mathrm{AC}$ & IB-III & 326 & & 326 & & & $9.75 \%$ & IB 7.69\%; IIA 0\%; IIB 15.38\% & \\
\hline Naoyuki et al. [4] & 1990 & Japan & $\mathrm{SCC}, \mathrm{AC}$ & IB-IIIB & 597 & & 597 & $0.19 \%$ & & $5.56 \%$ & & \\
\hline Sutton et al. [11] & 1992 & India & $\mathrm{SCC}, \mathrm{AC}$ & IB & 990 & & 990 & $0.52 \%$ & IB $0.52 \%$ & $1.65 \%$ & IA $0 \%$; IB $1.65 \%$ & \\
\hline Yamamoto et al. [5] & 2001 & Japan & $\mathrm{SCC}, \mathrm{AC}$ & IB-II & 631 & & 631 & $0.41 \%$ & IB $0 \%$; IIA $0 \%$; IIB $0.6 \%$ & $8.22 \%$ & IA $0 \%$; IB $2 \%$; IIA $0 \%$; IIB $16.22 \%$ & \\
\hline Nakanishi et al. [6] & 2001 & Japan & $\mathrm{SCC}, \mathrm{AC}$ & IA-IIB & 1304 & & 1304 & $1.32 \%$ & IIB $4.46 \%$ & $6.25 \%$ & IIB $22.22 \%$ & \\
\hline Shimada et al. [7] & 2005 & Japan & $\mathrm{SCC}, \mathrm{AC}$ & IB-IIB & 3471 & & 3471 & $0.80 \%$ & IB $0.22 \%$; IIA $0.75 \%$; IIB $2.02 \%$ & $5.31 \%$ & IB 3.72\%; IIA 5.26\%; IIB 9.85\% & \\
\hline Landoni et al. [14] & 2007 & Italy & $\mathrm{SCC}, \mathrm{AC}$ & IA2-IIA & 1965 & 1695 & 270 & $0.55 \%$ & & $2.37 \%$ & & \\
\hline Kim et al. [8] & 2007 & Korea & $\mathrm{SCC}, \mathrm{AC}$ & IA1-IIB & 625 & & 625 & $0.42 \%$ & & $7.95 \%$ & & \\
\hline Kasamatsu et al. [9] & 2009 & Japan & $\mathrm{SCC}, \mathrm{AC}$ & I-IIB & 578 & & 578 & $0.13 \%$ & IB $0.36 \%$; IIA $1.96 \%$; IIB $3.1 \%$ & $4.92 \%$ & IB $3.16 \%$; IIA 0\%; IIB 13.64\% & \\
\hline Hu et al. [10] & 2013 & China & $\mathrm{SCC}, \mathrm{AC}$ & IB-IIB & 1889 & & 1889 & $0.74 \%$ & IB $0.47 \%$; IIA $0.8 \%$; IIB $1.46 \%$ & $6.94 \%$ & IB 0.77\%; IIA 7.69\%; IIB 7.14\% & \\
\hline Matsuo et al. [15] & 2017 & Japan & $\mathrm{SCC}, \mathrm{AC}$ & IB-IIB & 5697 & & 5697 & $0.73 \%$ & & $2.56 \%$ & & \\
\hline Cao et al. [16] & 2019 & China & $\mathrm{SCC}, \mathrm{AC}$ & IA2-IIA2 & 5181 & 1496 & 3685 & $0.50 \%$ & & $3.07 \%$ & & \\
\hline Hopkins et al. [17] & 1986 & USA & $\mathrm{AC}$ & I-IV & 84 & 8 & & & & & & \\
\hline Lyu et al. [18] & 2014 & China & $\mathrm{AC}$ & I & 1639 & 577 & 1062 & & & & & \\
\hline Chen et al. [19] & 2016 & China & $\mathrm{AC}$ & IIB & 159 & 33 & 126 & & & $3.47 \%$ & IA $0 \%$; IB $2 \%$; IIA $7.69 \%$; IIB $5.56 \%$ & \\
\hline Hu et al. [20] & 2017 & China & $\mathrm{AC}$ & IIB & 105 & 19 & 86 & & & $2.86 \%$ & IA $0 \%$; IB $1.47 \%$; IIA $0 \%$; IIB $16.67 \%$ & \\
\hline Xie et al. [21] & 2017 & China & $\mathrm{AC}$ & IIA & 128 & 15 & 113 & $1.32 \%$ & & $0.08 \%$ & & \\
\hline Xu et al. [22] & 2018 & China & $\mathrm{AC}$ & I & 1386 & 278 & 1090 & & & & & \\
\hline Natsume et al. [23] & 1999 & Japan & $\mathrm{AC}$ & IB-II & 82 & & 82 & & & $12.90 \%$ & IB 3.22\%; IIA 33.3\%; IIB 21.43\% & \\
\hline Lu et al. [25] & 2017 & China & $\mathrm{AC}$ & IA2-IIA2 & 101 & & 101 & & & $4.95 \%$ & IA $0 \%$; IB $4.55 \%$; IIA $8.33 \%$; & Grade, LVSI, LMN, tumor size, DSI, UCI \\
\hline Zhou et al. [24] & 2012 & China & $\mathrm{AC}$ & I-IIB & 312 & & 312 & & & $4.50 \%$ & IB $2.3 \%$; IIA $10.81 \%$; IIB $8.33 \%$ & UCI, PMI, vaginal infiltration \\
\hline
\end{tabular}


Table 2. Quality assessment of included studies.

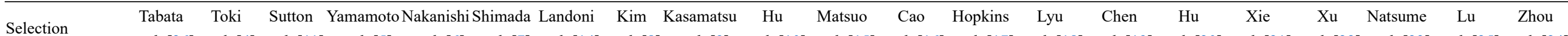
et al. [26] et al. [4] et al. [11] et al. [5] et al. [6] et al. [7] et al. [14] et al. [8] et al. [9] et al. [10] et al. [15] et al. [16] et al. [17] et al. [18] et al. [19] et al. [20]et al. [21] et al. [22] et al. [23] et al. [25]et al. [24]

Case definition

with independent

validation

Consecutive or

obviously repre-

sentative series of

cases

community con-

trols

No endpoint of

disease in con-

trols at start study

Comparability

Study controls for

age

Study controls for

FIGO stage

Exposure

Ascertainment of

exposure from se-

cure record

Same method and

ascertainment for

cases and controls

Same non-

response rate for

both groups

Total score

7

$8 \quad 9$




\section{Discussion}

This study has a large sample size for the comparison of the incidence of OM between AC and SCC. The overall incidence of $\mathrm{AC}$ with $\mathrm{OM}$ was $3.85 \%$, which was higher than that of SCC $(0.68 \%)$. The incidence of $\mathrm{OM}$ for $\mathrm{AC}$ was higher than that of SCC in stage IA-IIB (OR 5.68, 95\% CI $4.40-7.32, I^{2}=28.1 \%$ ). In the subgroup meta-analysis of stage I and II, we reached the same conclusion.

We found that the incidence of OM for SCC and AC were both $0 \%$ in stage IA so ovarian preservation is appropriate in this group. The incidence of OM for stage IB AC and SCC were not very high $(2.73 \%$ vs $0.8 \%)$ but The incidence of OM for stage IB AC was still higher than SCC. As a result, we suggest that ovaries should be preserved for stage IB patients without risk factors. The incidence of OM for stage IIA and IIB AC was as high as $5.95 \%$ and $12.86 \%$, so we do not recommend that patients with stage IIA and IIB AC retain their ovaries. Through meta-analysis, Hongyan Cheng et al. [27] believed that ovarian preservation was not recommended for stage IIB, while ovarian preservation for stage I-IIA was reasonable. Among the included studies, the incidence of OM for stage IIA was $3.4 \%$ and that for stage IIB was $11.8 \%$. Their sample size was smaller than ours, so there was a difference in the incidence of OM for stage IIA. Their meta-analysis did not separately regard stage IIA and IIB as risk factors.

In the meta-analysis to study the overall survival of ovarian preservation, we only included studies that specifically focused on the overall survival of ovarian preservation for $\mathrm{AC}$, and excluded studies when pathological data for $\mathrm{AC}$ was unavailable, as in the study performed by Matsuo [15]. Xie et al. [21] found that there was no difference in the 5 -year survival rate between patients with ovarian preservation and patients with oophorectomy (75\% vs $86.6 \%$; $p$ $>0.05$ ) for AC in t stage IB-IIA. For T1N0M0 cervical adenocarcinoma, Xu et al. [22] found that oophorectomy group had worse cause-specific survival (5-year $97.1 \%$ vs $98.8 \%, 10$-year $95.2 \%$ vs $98.0 \%, p=0.0370)$ and overall survival (5-year $97.1 \%$ vs $98.8 \%$, 10-year $93.5 \%$ vs $96.5 \%$, $p=0.0025$ ). Our meta-analysis found that ovarian preservation was not associated with statistically significant OS. In the sub-group analysis for stage I, we reached similar conclusions. In the existing literature, there is no data for OS of ovarian preservation especially for stage II AC. Due to lack of data, we did not perform a subgroup analysis of stage II. Therefore, whether patients with stage IIA and IIB could undergo ovarian preservation requires further research.

In the meta-analysis of the risk factors for $\mathrm{OM}$ in $\mathrm{AC}$, we only included studies on the risk factors of $\mathrm{OM}$ for $\mathrm{AC}$ and excluded studies that analyzed the risk factors of OM for cervical cancer where the pathologic data of $\mathrm{AC}$ was unavailable, such as the studies performed by Yamamoto, Min-Jeong Kim, and Ting Hu, Le Zhou $[5,8,10]$. A meta-analysis performed by Chen et al. [19] believed that stage IIB, deep stromal invasion, corpus uteri invasion and
Table 3. Selection criteria for ovarian preservation in patients with adenocarcinoma of the cervix.

Preoperative factors
Desire to preserve the ovaries
FIGO $\leq \mathrm{IB} 2$
No corpus uteri invasion (CT)
No deep stromal invasion (biopsy and cervical conization)
Tumor size $<4 \mathrm{~cm}$
No lympho-vascular space invasion (biopsy and cervical conization)
Intraoperative factors
Normal ovarian appearance
No evidence of extra-uterine spread

parametrial invasion were risk factors for $\mathrm{OM}$ in $\mathrm{AC}$. Our meta-analysis found that IIA was also a risk factor. A systemic review by Touhami concluded that age $>45$, FIGO $>$ stage IB, deep stromal invasion, lympho-vascular space invasion, corpus invasion, parametrial invasion and tumor size $>4 \mathrm{~cm}$ were risk factors [28]. Our meta-analysis found that age $>45\left(\right.$ OR $0.95,95 \%$ CI $\left.0.46-1.97, I^{2}=0 \%\right)$ and tumor grade (OR 0.93,95\% CI 0.4-2.18, $I^{2}=0 \%$ ) were not the risk factors for OM in early stage AC.

Our meta-analysis determined deep stromal invasion, tumor size $>4 \mathrm{~cm}$ and lympho-vascular space invasion were risk factor for $\mathrm{OM}$ in AC. According to NCCN guidelines, deep stromal invasion, tumor size $>4 \mathrm{~cm}$ and lymphovascular space invasion were also intermediate risk factors for pelvic recurrence [29]. Besides FIGO IIB, we also found FIGO IIA a risk factor for OM. Gynecological examination and CT before operation are useful to evaluate vaginal and parametrial involvement.

Finally, we established criteria for ovarian preservation in AC. The preoperative factors were as follows: desire to preserve the ovaries, no corpus uteri invasion (CT), no deep stromal invasion (cervical conization), tumor size $<4 \mathrm{~cm}$, FIGO stage $\leq$ IB2 (FIGO 2018), and no lymphovascular space invasion (biopsy and cervical conization). The intraoperative factors were as follows: normal ovarian appearance and no evidence for extra-uterine spread (Table 3).

Regarding the limitations of this study, we only included retrospective studies. The standard of ovarian preservation for $\mathrm{AC}$ needs to be further verified by prospective studies. The overall survival analysis was limited by small numbers. Given it was only limited to stage I disease, it is difficult to make a definitive conclusion.

\section{Conclusions}

Ovarian preservation is only recommended in stage IA and stage IB AC without risk factors, but it is not reasonable for stage IIA and IIB AC. Both stage IIA and IIB are risk factors for $\mathrm{OM}$ in early stage $\mathrm{AC}$. 


\section{Author contributions}

DW-Investigation, Formal analysis, Resources, Manuscript writing. LZ-Data collection. NBSupervision, Validation, Manuscript editing. LYData collection. YNW-Investigation, Data collection. YCW-Data analysis. MML_-Manuscript editing. JLManuscript editing. JW-Funding acquisition, Project development. All authors read and approved the final manuscript.

\section{Ethics approval and consent to participate}

Not applicable.

\section{Acknowledgment}

Not applicable.

\section{Funding}

This study was supported by Jiangsu Provincial Medical Talent (2016KJQWZDRC-02).

\section{Conflict of interest}

The authors declare no conflict of interest.

\section{References}

[1] Chung HH, Jang MJ, Jung KW, Won YJ, Shin HR, Kim JW, et al. Cervical cancer incidence and survival in Korea: 1993-2002. International Journal of Gynecological Cancer. 2006; 16: 1833-1838.

[2] Smith HO, Tiffany MF, Qualls CR, Key CR, et al. The rising incidence of adenocarcinoma relative to squamous cell carcinoma of the uterine cervix in the United States: a 24-year population-based study. Gynecologic Oncology. 2000; 78: 97-105.

[3] Davy MLJ, Dodd TJ, Luke CG, Roder DM, et al. Cervical cancer: effect of glandular cell type on prognosis, treatment, and survival. Obstetrics and Gynecology. 2003; 101: 38-45.

[4] Toki N, Tsukamoto N, Kaku T, Toh N, Saito T, Kamura T, et al. Microscopic ovarian metastasis of the uterine cervical cancer. Gynecologic Oncology. 1991; 41: 46-51.

[5] Yamamoto R, Okamoto K, Yukiharu T, Kaneuchi M, Negishi H, Sakuragi N, et al. A Study of Risk Factors for Ovarian Metastases in Stage IB-IIIB Cervical Carcinoma and Analysis of Ovarian Function after a Transposition. Gynecologic Oncology. 2001; 82: 312316.

[6] Nakanishi T, Wakai K, Ishikawa H, Nawa A, Suzuki Y, Nakamura S, et al. A Comparison of Ovarian Metastasis between Squamous Cell Carcinoma and Adenocarcinoma of the Uterine Cervix. Gynecologic Oncology. 2001; 82: 504-509.

[7] Shimada M, Kigawa J, Nishimura R, Yamaguchi S, Kuzuya K, Nakanishi $\mathrm{T}$, et al. Ovarian metastasis in carcinoma of the uterine cervix. Gynecologic Oncology. 2006; 101: 234-237.

[8] Kim M, Chung HH, Kim JW, Park N, Song Y, Kang S, et al. Uterine corpus involvement as well as histologic type is an independent predictor of ovarian metastasis in uterine cervical cancer. Journal of Gynecologic Oncology. 2008; 19: 181-184.

[9] Kasamatsu T, Onda T, Sawada M, Kato T, Ikeda S, Sasajima Y, et al. Radical hysterectomy for FIGO stage I-IIB adenocarcinoma of the uterine cervix. British Journal of Cancer. 2009; 100: 1400-1405.

[10] Hu T, Wu L, Xing H, Yang R, Li X, Huang K, et al. Development of Criteria for Ovarian Preservation in Cervical Cancer Patients Treated with Radical Surgery with or without Neoadjuvant Chemotherapy: a Multicenter Retrospective Study and Meta-analysis. Annals of Surgical Oncology. 2013; 20: 881-890.
[11] Sutton GP, Bundy BN, Delgado G, Sevin BU, Creasman WT, Major $\mathrm{FJ}$, et al. Ovarian metastases in stage IB carcinoma of the cervix: a Gynecologic Oncology Group study. American Journal of Obstetrics and Gynecology. 1992; 166: 50-53.

[12] Rocca WA, Grossardt BR, de Andrade M, Malkasian GD, Melton $\mathrm{LJ}$, et al. Survival patterns after oophorectomy in premenopausal women: a population-based cohort study. The Lancet Oncology. 2006; 7: 821-828.

[13] Parker WH, Broder MS, Chang E, Feskanich D, Farquhar C, Liu Z, et al. Ovarian conservation at the time of hysterectomy and longterm health outcomes in the nurses' health study. Obstetrics and Gynecology. 2009; 113: 1027-1037.

[14] Landoni F, Zanagnolo V, Lovato-Diaz L, Maneo A, Rossi R, Gadducci A, et al. Ovarian metastases in early-stage cervical cancer (IA2-IIA): a multicenter retrospective study of 1965 patients (a Cooperative Task Force study). International Journal of Gynecological Cancer. 2007; 17: 623-628.

[15] Matsuo K, Shimada M, Yamaguchi S, Kanao H, Nakanishi T, Saito $\mathrm{T}$, et al. Identifying a candidate population for ovarian conservation in young women with clinical stage IB-IIB cervical cancer. International Journal of Cancer. 2018; 142: 1022-1032.

[16] Cao L, Wen H, Feng Z, Han X, Wu X, et al. Distinctive clinicopathologic characteristics and prognosis for different histologic subtypes of early cervical cancer. International Journal of Gynecologic Cancer. 2019; 29: 1244-1251.

[17] Hopkins MP, Sutton P, Roberts JA. Prognostic features and treatment of endocervical adenocarcinoma of the cervix. Gynecologic Oncology. 1987; 27: 69-75.

[18] Lyu J, Sun T, Tan X. Ovarian preservation in young patients with stage I cervical adenocarcinoma: a surveillance, epidemiology, and end results study. International Journal of Gynecological Cancer. 2014; 24: 1513-1520.

[19] Chen J, Wang R, Zhang B, Lin X, Wei J, Jia Y, et al. Safety of ovarian preservation in women with stage $i$ and II cervical adenocarcinoma: a retrospective study and meta-analysis. American Journal of Obstetrics and Gynecology. 2016; 215: 460.e1-460.e13.

[20] Hu J, Jiao X, Yang Z, Cui H, Guo H, Wu Y, et al. Should ovaries be removed or not in early-stage cervical adenocarcinoma: a multicenter retrospective study of 105 patients. Journal of Obstetrics and Gynaecology. 2017; 37: 1065-1069.

[21] Xie X, Song K, Cui B, Jiang J, Yang X, Kong B, et al. A comparison of the prognosis between adenocarcinoma and squamous cell carcinoma in stage IB-IIA cervical cancer. International Journal of Clinical Oncology. 2018; 23: 522-531.

[22] Xu H, Tang X, Ding J, Qiu J, Zhang X, Hua K, et al. Ovarian conservation is associated with better survival in young patients with T1N0M0 cervical adenocarcinoma: a population-based study. Archives of Gynecology and Obstetrics. 2018; 297: 775-784.

[23] Natsume N, Aoki Y, Kase H, Kashima K, Sugaya S, Tanaka K, et al. Ovarian metastasis in stage IB and II cervical adenocarcinoma. Gynecologic Oncology. 1999; 74: 255-258.

[24] Zhou J, Chen Y, Zhang P, Lou H. Ovarian preservation in adenocarcinoma of the uterine cervix. Journal of Ovarian Research. 2017; 10: 48 .

[25] Lu H, Li J, Wang L, Zhou H, Liu Y, Wang D, et al. Is Ovarian Preservation Feasible in Early-Stage Adenocarcinoma of the Cervix? Medical Science Monitor. 2016; 22: 408-414.

[26] Tabata M, Ichinoe K, Sakuragi N, Shiina Y, Yamaguchi T, Mabuchi $\mathrm{Y}$, et al. Incidence of ovarian metastasis in patients with cancer of the uterine cervix. Gynecologic Oncology. 1987; 28: 255-261.

[27] Cheng HY, Huo LQ, Zong LJ, Kong Y, Yang J, Xiang Y, et al. Oncological Outcomes and Safety of Ovarian Preservation for Early Stage Adenocarcinoma of Cervix: a Systematic Review and MetaAnalysis. Frontiers in Oncology. 2019; 9: 777.

[28] Touhami O, Plante M. Should ovaries be removed or not in (earlystage) adenocarcinoma of the uterine cervix: A review. Gynecologic Oncology. 2015; 136: 384-388.

[29] Nadeem R, Catheryn M, Sarah B, Kristin B, Susana MC, Hye SC, et al. NCCN Clinical Practice Guidelines in Oncology Cervical Cancer Version 1. 2021. Available at: https://www.ncen.org/home (Accessed: 27 November 2021). 